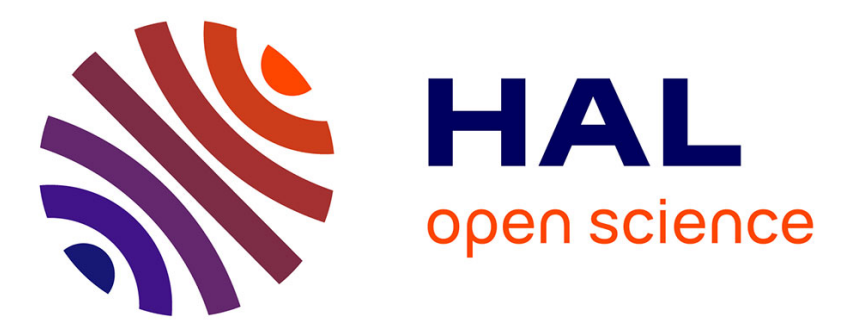

\title{
Charge and spin fluctuations in the Hubbard model: Fermi liquid properties at low temperature
}

M. Lavagna

\section{To cite this version:}

M. Lavagna. Charge and spin fluctuations in the Hubbard model: Fermi liquid properties at low temperature. International Journal of Modern Physics B, 1991, 05 (6-7), pp.885-905. 10.1142/S021797929100047X . hal-01896292

\section{HAL Id: hal-01896292 \\ https://hal.science/hal-01896292}

Submitted on 17 Oct 2018

HAL is a multi-disciplinary open access archive for the deposit and dissemination of scientific research documents, whether they are published or not. The documents may come from teaching and research institutions in France or abroad, or from public or private research centers.
L'archive ouverte pluridisciplinaire HAL, est destinée au dépôt et à la diffusion de documents scientifiques de niveau recherche, publiés ou non, émanant des établissements d'enseignement et de recherche français ou étrangers, des laboratoires publics ou privés. 


\title{
CHARGE AND SPIN FLUCTUATIONS IN THE HUBBARD MODEL: FERMI LIQUID PROPERTIES AT LOW TEMPERATURES
}

\author{
M. LAVAGNA \\ Institut Laue-Langevin, 156X, 38042 Grenoble Cedex, France
}

\begin{abstract}
We review the slave-boson representations of the Hubbard model. When both spin and charge fermion degrees of freedom are represented by Bose fields, the approach is equivalent at the "self-consistent" saddle point level to the Gutzwiller approximation (GA). We show how the determination of the Lagrange multiplicrs, introduced to enforce the constraints, involves a Mott-Hubbard gap near half-filling for $U>U_{\mathrm{c}}$ (localization edge). The quantum fluctuations are then considered within a renormalized basis of boson fields (restoring the spin-rotation invariance), which brings out two distinct channels, symmetric and antisymmetric. It is remarkable that both spin and charge fluctuations are obtained at the same level of the gaussian fluctuations, as oppossed to the standard $1 / N$ expansion. This provides the microscopic basis for a Fermi liquid theory. Both dynamic effects and extension to finite temperature which were beyond the scope of the variational procedurc can now be described by this approach. The physical implications of the model (dynamic correlation functions, $T^{3} \mathrm{Ln} T$ term of the specific heat, superfluid instability) are finally considered, all of which reflect the Fermi liquid nature of the system.
\end{abstract}

The study of strongly-correlated systems $\left({ }^{3} \mathrm{He},{ }^{1}\right.$ heavy fermions, ${ }^{2}$ quantum Hall effect, ${ }^{3}$ and more recently high-temperature superconductors ${ }^{4} \ldots$ ) has led to much research to develop new methods to attack the problem of correlations inherent in ail these systems of fermions characterized by strong on-site repulsions. We present here one of these methods, the slave-boson approach. Originally introduced in the context of the single-Kondo impurity problem, ${ }^{5,6}$ it has shown itself to be very powerful in the study of heavy fermions ${ }^{7-9}$ (Anderson lattice). Its more recent adaptation to the Hubbard model appears to have already given by very encouraging results.

The approach is actually related - though this was realized much later ${ }^{10}-$ to the variational method introduced earlier on by Gutzwiller ${ }^{11.12}$ (see also Refs. 13 and 14 for a very good review). The ansatz introduced for the $N$-particle wave function (Gutzwiller wave function GWF) consists of a Slater determinant for the electrons, together with a projector (primitive version of a Jastrow factor) whose effect is to reduce the weight of configurations containing doubly occupied sites, by an amount to be variationally determined.

Although the procedure is well established, the calculations turned out to be intractable, and Gutzwiller introduced a quasi-classical approximation. It has the advantage of allowing a simple formulation of the problem which permits to bring to the foreground of the essential physical aspects: 
(i) The main one is the localization obtained at half-filling for strong coupling. ${ }^{15}$ This localization due to the effect of correlations is related to the general phenomena of the Mott transition.

(ii) The second aspect is the magnetism with, for example, the occurrence of an antiferromagnetic instability close to half-filling for any alternated structure.

The success of the Gutzwiller approximation (GA) is to permit a description of the Fermi liquid at low temperature, thus providing a microscopic basis for the Landau theory. The precision with which it describes the properties of the normal phase of liquid $\mathrm{He}^{3}$ (the dependence of $F_{0}^{s}$ and $F_{0}^{a}$ on the pressure, ${ }^{13}$ for example) is quite extraordinary and confirms the foundation of the "almost" localized model, as opposed to the "almost" magnetic picture provided by a theory of paramagnons. ${ }^{16,17}$

It is only recently that the Gutzwiller ansatz could be treated exactly in some limits without requiring the Gutzwiller approximation. ${ }^{18,19}$ When the dimensionality $d$ tends to infinity, ansatz and approximation coincide, while for $d=1$, the results produced by the ansatz are in good agreement with the exact results obtained from the Bethe ansatz. This agreement becomes complete $\mathrm{e}^{20,21}$ in the case of the Heisenberg model with an interaction $J_{i j} \sim\left(r_{i j}\right)^{-2}$.

Apart from these two limiting cases, the Gutzwiller ansatz is not exactly soluble, and the Gutzwiller approximation can still be regarded as a very interesting starting point from which one may imagine developing a more systematic theory to correct the weaknesses of the GA. Recall that these weaknesses are inherent in any mean-field theory. It fails to describe the dynamic effects, particularly the motion of holes in the almost localized regime and has no correct generalization to finite temperatures. Hence the idea is to incorporate the dynamic effects missed by the approximation, and hope to combine the motion of dynamic fluctuations RPA type with the "almost" localized model provided by the Gutzwiller approximation. This project was inaccessible in the framework of the variational formulation. It has recently become realizable $\mathrm{e}^{22-24}$ in the framework of the slave-boson representation introduced by Kotliar-Ruckenstein, which allowed one to transform the variational procedure into a standard perturbative procedure, the GA being introduced at the first level of the saddle-point approximation in its selfconsistent version.

In Sec. 1, we will present the slave-boson representation of the Hubbard model as introduced in Ref. 10. The problem involved is typical of a constrained system since the projection on the physical subspace requires the introduction of some constraints: these are enforced by the standard methods of introducing Lagrange multipliers. As pointed out by Li et al., ${ }^{25}$ the initial representation breaks the spin-rotation invariance (SRI) which is then restored by considering a generalized SRI representation. We will end this first section by making some comparisons with other choices of representations such as the $U=\infty$ slave-boson representation of the generalized degeneracy $N=\infty$ Hubbard model, or the Schwinger-boson representation which has proved to be very useful for the study of the Heisenberg 
model. In Sec. 2, we will review the simplest saddle-point approximation available where the boson fields are taken as space- and time-independent. In its "selfconsistent" version (with an implicit dependence of the boson fields on the external excitation), this is equivalent to the Gutzwiller approximation. For example, it defines mass-enhanced quasiparticles with a metal-insulator transition at half-filling for strong enough coupling. The new information contained in the slave-boson approach (as compared with the GA) concerns the determination of the Lagrange multipliers at the saddle-point. As remarked by the author, ${ }^{24}$ these are marked by a splitting on each side of the localization transition line, which can be interpreted as a Mott-Hubbard gap. Section 3 is devoted to the derivation of the quantum fluctuations. ${ }^{22.24}$ It is remarkable that both the charge fluctuations (bubble diagram) and spin fluctuations (ladder diagram) arise at the same level of the gaussian fluctuations. We will argue that this constitutes a decisive progress compared to the $1 / N$ expansions ${ }^{27}$ since now the spin-fluctuations, so important to describe the magnetic properties of real systems, are already described at the same level as the charge fluctuations. This defines effective interactions among quasi-particles respectively in symmetric and antisymmetric channels, and generalizes the Fermi liquid description provided by the Gutzwiller approximation to any channel $l \neq 0$. It restores the dynamic effects which were missed in the GA, and solves the delicate question concerning the extension to finite temperature. ${ }^{28}$ In Sec. 4, we will review the physical implications of the model, all of them being typical of a Fermi-liquid: dynamic correlation functions, $T^{3} \mathrm{Ln} T$ contribution to the specific heat, superfluid (or superconducting) instability ....

\section{Slave-Boson Representation of the Hubbard Model}

Our starting point is the Hubbard model for interacting electrons on a lattice:

$$
H=-\sum_{i j \sigma} t_{i j} f_{i \sigma}^{+} f_{i \sigma}+U \sum f_{i \sigma}^{+} f_{i \sigma} f_{i-\sigma}^{+} f_{i-\sigma}
$$

where $f_{1 \sigma}^{+}\left(f_{i \sigma}\right)$ are the creation (annihilation) operators of an electron with spin projection $\sigma(= \pm 1)$ in an atomic state at site $i, t_{i j}\left(=t_{j i}\right)$ is the hopping matrix element for two electrons of opposite spins at the same site.

There are four atomic states per site $i,\left|O_{i}\right\rangle,\left|\uparrow_{t}\right\rangle,\left|\downarrow_{i}\right\rangle,\left|\uparrow \downarrow_{i}\right\rangle$, corresponding to the empty site, the singly occupied site with electron spin polarization $\uparrow$ or $\downarrow$, and the doubly occupied site. In the spirit of the Gutzwiller solution, Kotliar and Ruckenstein ${ }^{10}$ proposed to keep track of the local configurations by introducing some additional degrees of liberty represented by four fields $e_{i}, p_{i \sigma}$ and $d_{i}$. The corresponding occupation numbers $e_{i}^{+} e_{i}, p_{i \sigma}^{+} p_{i \sigma}$ and $d_{i}^{+} d_{i}$ represent the projectors on the four possible states on site $i$. This turns out to replace the Hilbert space of fermions states by an enlarged Hilbert space of fermion and boson states whose correspondence is given by: 
Initial representation

New representation

$$
\begin{array}{lll}
\left|O_{i}\right\rangle=|\mathrm{vac}\rangle & \rightarrow & |0\rangle \\
\left|\uparrow_{i}\right\rangle=f_{i \uparrow}^{+}|\mathrm{vac}\rangle & \rightarrow & p_{i \dagger}^{+} e_{i} c_{i \uparrow}^{+}|0\rangle \\
\left|\downarrow_{i}\right\rangle=f_{i \downarrow}^{+}|\mathrm{vac}\rangle & \rightarrow & p_{i \dagger}^{+} e_{i} c_{i \dagger}^{+}|0\rangle \\
\left|\uparrow \downarrow_{i}\right\rangle=f_{i \uparrow}^{+} f_{i \downarrow}^{+}|\mathrm{vac}\rangle & \rightarrow & d_{i \uparrow}^{+} e_{i} c_{i \dagger}^{+} c_{i \downarrow}^{+}|0\rangle . \\
& & \text { where }|0\rangle=e_{i}^{+}|\mathrm{vac}\rangle .
\end{array}
$$

The two representations are equivalent provided that some constraints are satisfied which guarantee the physical meaning of the fields:

$$
\begin{aligned}
& P_{i}=e_{i}^{+} e_{i}+\sum_{\sigma} p_{i \sigma}^{+} p_{i \sigma}+d_{i}^{+} d_{i}-1=0 \\
& \text { (completeness of the projectors), } \\
& Q_{i \sigma}=c_{i \sigma}^{+} c_{1 \sigma}-p_{i \sigma}^{+} p_{i \sigma}-d_{i}^{+} d_{i}=0 \quad \forall \sigma \\
& \text { (redundancy between } c_{\sigma} \text { and } p_{o}, d \text { degrees of liberty). }
\end{aligned}
$$

The constraint (1.2) automatically implies that the operators $n_{\alpha}=\alpha^{+} \alpha$ (where $\alpha=e, p_{\sigma}$ or $d$ ) satisfy the algebra of projectors:

$$
n_{\alpha} n_{\beta}=\delta_{\alpha \beta} n_{\alpha} .
$$

In order to obtain the expression of the Hubbard Hamiltonian in the new representation, let us establish the following correspondence between operators (easy to prove matrically):

$$
\begin{gathered}
f_{i \sigma}^{+} f_{j \sigma} \rightarrow \bar{z}_{i \sigma}^{+} \bar{z}_{j \sigma} c_{i \sigma}^{+} c_{j \sigma} \text { for } i \neq j \text { with } \bar{z}_{i \sigma}=e_{i}^{+} p_{i \sigma}+p_{i-\sigma}^{+} d_{i}, \\
\qquad n_{i \uparrow} n_{i \downarrow} \rightarrow d_{i}^{+} d_{i}^{+} .
\end{gathered}
$$

Then the expression of the Hubbard Hamiltonian in the new representation can be written:

$$
H=-\sum_{i j \sigma} t_{i j} \bar{z}_{i \sigma}^{+} \bar{z}_{j \sigma} c_{i \sigma}^{+} c_{j \sigma}+U \sum_{i} d_{i}^{+} d_{i},
$$

as long as the constraints are satisfied.

Let us make three remarks:

(i) To guarantee the fermionic character of the physical particle, the operators which represent it in the second quantization must respect the usual 
anti-commutation rules. This is automatically insured if $c_{i \sigma}$ obey Fermi statistics, and $e_{i} p_{i \sigma}, d_{i}$ Bose statistics (hence the term of "slave-boson").

(ii) Indeed, the choice $\bar{z}_{i \sigma}$ is not unique, but one can replace $\bar{z}_{i \sigma}$ by any combination $U_{i \sigma} \overline{\bar{z}}_{i \sigma} V_{i \sigma}$, where $U_{i \sigma}$ and $V_{i \sigma}$ are diagonal matrices whose only non-zero term (equal to 1) appears respectively on $|0\rangle,|\sigma\rangle,|-\sigma\rangle,|\uparrow\rangle$ lines. One can in particular choose as in Ref. 10:

$$
z_{l \sigma}=\left(1-d_{i}^{+} d_{l}-p_{l \sigma}^{+} p_{i \sigma}\right)^{-1 / 2} \bar{z}_{l \sigma}\left(1-e_{i}^{+} e_{i}-p_{i-\sigma}^{+} p_{i-\sigma}\right)^{-1 / 2} .
$$

All the choices are formally equivalent so far as the constraints are exactly satisfied. This is no longer true when one makes approximations. Typically, the mean-field approximation satisfies the constraint on average, and leads to different results depending on the special choice of $z_{i \sigma}$. These discrepancies are supposed to disappear if one could include quantum fluctuations at all orders. When stopping at mean-field, the choice (1.6) is more sensible since it renormalizes $\bar{z}_{i \sigma}$ by its value in the uncorrelated case and gives back the free electron gas in the limit $U=0$. It is this choice that will be retained in this paper.

(iii) In fact, the original slave-boson representation breaks the spin-rotation invariance coming from the nature of the boson operators $p_{i \sigma}$. To avoid the difficulties which may arise from this, $\mathrm{Li}$ et $a l^{25}$ introduced a generalized spin rotation invariant (SRI) representation which automatically preserves this symmetry. They did this by introducing two-component matrix operators $\tilde{p}_{i}=\frac{1}{\sqrt{2}} \sum_{\alpha=0}^{4} p_{i \alpha} \cdot \tilde{\tau}_{\alpha}$ where $\tilde{\tau}_{\alpha}$ are the Pauli matrices. This introduces a scalar boson $p_{i 0}(\operatorname{spin} 0)$ and three-component vector boson $\mathbf{p}_{i}(\operatorname{spin} 1)$ which obey the usual commutation rule:

$$
\left[p_{i \alpha}, p_{j \beta}^{+}\right]=\delta_{i j} \delta_{\alpha \beta} \text {. }
$$

Then automatically the state $|\sigma i\rangle$ whose SRI expression is $\sum_{\sigma^{\prime}} p_{i \sigma \sigma^{\prime}}^{+} e_{i} c_{i \sigma^{\prime}}^{+}|0\rangle$ transforms as a spinor.

As expected, the spin-1/2 character of the state $\left|\sigma_{i}\right\rangle$ imposes the condition that $p_{i}$ might be either of spin 0 or 1 , hence the tensor nature of the operator $\tilde{p}_{i}$. The formal Kotliar-Ruckenstein representation can be viewed as a particular choice of the generalized representation corresponding to a diagonalized formulation whose only non-zero terms are $p_{i \uparrow}$ and $p_{i \downarrow}$.

To end this section, we will mention the possibility of choosing other types of representations:

1. For the generalized $N=\infty$ Hubbard model and for an infinite $U$, it is enough to consider only one type of slave-boson, say $e$. This is closer to the initial formulation introduced by Coleman ${ }^{5}$ in the context of the Anderson model. In fact, it is easy to see that this representation coincides in the paramagnetic 
case, with the $U \rightarrow \infty, N \rightarrow \infty$ limit of a generalized $N$ Kotliar-Ruckenstein representation (where $d$ vanishes, and $p_{\sigma}$ cancels with the square root term at the denominator). However, we argue that this representation is much less powerful than the 4-slave-boson representation. The reason is that, although the saddle-point is exact at $N=\infty$, the $1 / N$ expansion term (gaussian fluctuations) only gives the charge fluctuations and misses the spin fluctuations so important to describe the magnetism. The following terms needed in the expansion (at least of order $1 / N^{2}$ ) lead to very tedious calculations. ${ }^{29}$ As we will see below, the 4-slave-boson representation has the huge advantage of describing charge and spin fluctuations at the same level of the gaussian fluctuations.

2. In the $U=\infty, N=\infty$ limit of the generalized Hubbard model, the creation operator of a particle is given by $e_{i} c_{i \sigma}^{+}$for any intersite term (hopping term), but by $c_{i \sigma}^{+}$for any on-site term (for example, the spin is represented by $\mathbf{c}_{i} \tau_{\alpha} \mathbf{c}_{i}$ ). To guarantee the fermionic character of the physical particle, one may define $c_{i \sigma}^{+}$as fermions (and $e_{i}^{+}$as bosons), or the opposite: $c_{i \sigma}^{+}$as bosons (and $e_{i}^{+}$as fermions). When put in the $J$-term, it turns out that this last definition is nothing but the Schwinger-boson representation of the spin. This has been intensively studied, first for the Heisenberg model ${ }^{30}$ and then for the $t-J$ model. ${ }^{31}$ It yields very interesting results at the saddle point level, with notably the formation of a Haldane gap at $d=1$ (for both integer and half-odd integer spins), and the existence of a Néel state at $d=2$.

\section{Saddle-Point Approximation}

The saddle-point approximation is independent of the choice of the gauge. So, for simplicity, we present it in the diagonalized KR gauge. The constraints may be incorporated by adding Lagrange multiplier terms. Since the time evolution generated by $H$ preserves the constraints $\left(\left[H, P_{i}\right]=\left[H, Q_{i \sigma}\right]=0\right)$, it is only necessary to enforce the constraint at one particular time, and consider Lagrange multipliers as time-independent.

The partition function can be written as a functional integral over the fermion and boson fields:

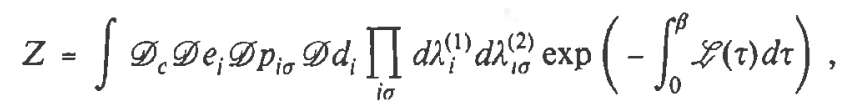

where the lagrangian $\mathscr{L}(\tau)$ is

$$
\mathscr{L}(\tau)=\mathscr{L}^{B}(\tau)+\mathscr{L}^{F}(\tau),
$$

where

$$
\mathscr{L}^{B}(\tau)=\sum_{i} e_{i}^{+}\left(\partial_{\tau}+\lambda_{i}^{(1)}\right) e_{i}+\sum_{i \sigma} p_{i \sigma}^{+}\left(\partial_{\tau}+\lambda_{i}^{(1)}-\lambda_{i \sigma}^{(2)}\right) p_{i \sigma}
$$




$$
\begin{gathered}
+\sum_{i} d_{i}^{+}\left(\partial_{\tau}+\lambda_{i}^{(1)}-\sum_{\sigma} \lambda_{i \sigma}^{(2)}+U\right) d_{i}-\lambda_{i}^{(1)}, \\
\mathscr{L}^{F}(\tau)=\sum_{i, \sigma} c_{l \sigma}^{+}\left[\left(\partial_{\tau}+\lambda_{i \sigma}^{(2)}\right) \delta_{i j}-t_{l j} z_{l \sigma}^{+} z_{j \sigma}\right] c_{j \sigma} .
\end{gathered}
$$

Integration over the Grassman variables yields ${ }^{10}$

$$
\begin{gathered}
Z=\int \mathscr{D} e_{i} \mathscr{D} p_{i \sigma} \mathscr{D} d_{i} \prod_{i \sigma} d \lambda_{i}^{(1)} d \lambda_{i \sigma}^{(2)} \exp \left(-\int_{0}^{\beta} \mathscr{L}_{\mathrm{eff}}^{(\tau)} d \tau\right), \\
\mathscr{L}_{\mathrm{eff}}(\tau)=\mathscr{L}_{(\tau)}^{B}+\operatorname{Tr} \cdot \operatorname{Ln}\left[\left(\delta_{\tau}+\lambda_{l \sigma}^{(2)}\right) \delta_{i j}+t_{i j} z_{i \sigma}^{+} z_{j \sigma}\right],
\end{gathered}
$$

where the trace has to be taken over position and spin variables. Kotliar and Ruckenstein ${ }^{10}$ proposed the simplest saddle-point approximation where the boson fields are considered as uniform and static. Then the free energy per site is

$$
F_{0}=f_{0}+U d_{0}^{2}+\lambda_{0}^{(1)}\left(e_{0}^{2}+\sum_{\sigma} p_{0 \sigma}^{2}+d_{0}^{2}-1\right)+\sum_{\sigma} \lambda_{0 \sigma}^{(2)}\left(n_{\sigma}-p_{0 \sigma}^{2}\right)
$$

with

$$
f_{0}=-k_{B} T \sum_{\sigma} \int_{-\infty}^{+\infty} \operatorname{Ln}\left(1+\exp -\beta q_{0 \sigma} \xi\right) \rho(\xi) d \xi
$$

where $\beta=1 / k_{B} T, q_{0 \sigma}=z_{0 \sigma}^{2}$, and $\rho(\varepsilon)$ is the density of states of the tight-binding band defined by $t_{i j}$.

At the saddle-point level, the constraints are satisfied on average and one immediately recognizes ${ }^{10}$ the result of the Gutzwiller approximation. At low temperature, the main contribution to the free energy $f_{0}$ comes from the energy

$$
E=2 \int \rho(\xi) q_{0} \xi n_{F}(q \xi) d \xi=q_{0}|\varepsilon|,
$$

where $|\varepsilon|$ is the averaged energy by site in the uncorrelated case. The values of the boson fields and Lagrange multipliers can be obtained by solving the saddle-point equations (minimization of the free energy with respect to the 7 parameters $\left.e_{0}, p_{0 \sigma}, d_{0}, \lambda_{0}^{(1)}, \lambda_{0 \pi}^{(2)}\right)$. As remarked by the author, ${ }^{24}$ this leads, in addition to the three constraints, to the following equation in the paramagnetic case (where the 0 indices are now omitted):

$$
\lambda^{(1)}=\frac{4 p^{2}}{1-\delta^{2}} \frac{x}{e}|\varepsilon|\left[1+\frac{2 e x}{1-\delta}\right] \text {, }
$$




$$
\begin{gathered}
U+\lambda^{(1)}-2 \lambda^{(2)}=\frac{4 p^{2}}{1-\delta^{2}} \frac{x}{d}|\varepsilon|\left[1+\frac{2 d x}{1+\delta}\right], \\
\lambda^{(1)}-\lambda^{(2)}=2 x^{2}|\varepsilon|\left[1+\frac{2 p^{2}}{1-\delta}+\frac{2 p^{2}}{1+\delta}\right],
\end{gathered}
$$

where we have used the convenient notations $s^{32} x=e+d . e, p, d$ (and $q$ ) can be expressed in terms of $x$ as

$$
\begin{aligned}
d^{2} & =\left(\frac{x^{2}-\delta}{2 x}\right)^{2}, \\
e^{2} & =\left(\frac{x^{2}+\delta}{2 x}\right)^{2}, \\
p^{2} & =\frac{2 x^{2}-x^{4}-\delta^{2}}{4 x^{2}}, \\
q & =\frac{2 x^{2}-x^{4}-\delta^{2}}{1-\delta^{2}} .
\end{aligned}
$$

By combining Eqs. (2.4), one can eliminate the Lagrange multipliers and obtain the usual optimization equation of the Gutzwiller approximation. This result is not surprising since it simply means

$$
d\left(f_{0}+U d_{0}^{2}\right) / d d_{0}^{2}=0,
$$

where $e_{0}$ and $p_{0}$ are expressed as a function of $d_{0}$ using the constraints. The resulting equation at $T=0$ is the following:

$$
\left(1-x^{2}\right) \frac{x^{4}}{x^{4}-\delta^{2}}=\tilde{u}
$$

with

$$
\tilde{u}=u \frac{1-\delta^{2}}{1-\alpha \delta^{2}}
$$

and

$$
u=\frac{U}{8\left|\varepsilon_{0}\right|},
$$

where $\left|\varepsilon_{0}\right|$ is the value of the energy $|\varepsilon|$ at half-filling and $\alpha$ is a parameter that characterizes the initial band of density $\rho_{0}: \alpha=1 /\left(4 \rho_{0}\left|\varepsilon_{0}\right|\right)=1$ for a rectangular 
band, and $3 \pi^{2} / 32=0.925$ for an elliptic one. One can, in principle, solve the system of equations (2.6) at any point of the diagram $(U, \delta)$. In fact, we will content ourselves with describing the most interesting region (in the sense of the correlations) around half-filling. A glance at the behavior of

$$
A(x)=\left(1-x^{2}\right) x^{4} /\left(x^{4}-\delta^{2}\right)
$$

enables us to distinguish two regimes:

Regime I (metallic regime): $\tilde{u} \simeq u<1, n=1$. Then

$$
\begin{aligned}
x^{2} & =1-u, \\
e^{2} & =d^{2}=(1-u) / 4, \\
p^{2} & =(1+u) / 4, \\
q & =1-u^{2} .
\end{aligned}
$$

Regime II (vacancy regime): $\tilde{u}=u>1, x \simeq \sqrt{\delta} \ll 1$. Then

$$
\begin{aligned}
x^{2} & =\delta / \zeta, \\
e^{2} & =\frac{\delta(1+\zeta)^{2}}{4 \zeta}, \\
p^{2} & =\frac{1}{2}, \\
q & =2 \delta / \zeta,
\end{aligned}
$$

with

$$
\zeta=\sqrt{1-U_{\mathrm{c}} / U} .
$$

One recovers the Brinkman-Rice ${ }^{15}$ transition (Mott localization) above a critical localization edge $U_{\mathrm{c}}=8\left|\varepsilon_{0}\right|$. Above $U_{\mathrm{c}}$, one needs to introduce some vacancies (or electrons) to restore the metallic behavior. This is conduction by vacancies. Note that, for infinite $U$, double occupation is strictly-forbidden $(d=0)$, while the bandwidth $\sim \delta$ is different from zero in the vacancy regime. Regime $\mathrm{I}\left(U<U_{\mathrm{c}}\right)$ is believed to provide a "lattice" description of normal ${ }^{3} \mathrm{He}$, and the new high $T_{\mathrm{c}}$ superconductors in their normal phase are supposed to be good candidates for regime II.

Indeed, the content of the saddle-point equations (2.4) goes beyond the Gutzwiller approximation, and also determines the values of the Lagrange multipliers at the saddle-point. One finds the following ${ }^{24}$. 
Regime I:

$$
\begin{gathered}
\lambda^{(1)}=\frac{U_{\mathrm{c}}}{4}(1+u)(2-u), \\
\lambda^{(2)}=\frac{U}{2} .
\end{gathered}
$$

Regime II:

$$
\begin{aligned}
& \lambda^{(1)}=\frac{U}{2}(1 \pm \zeta)\left[1+\frac{\delta}{\zeta}(1+\zeta)\right], \\
& \lambda^{(2)}=\frac{U}{2}(1 \pm \zeta)\left[1-\frac{\delta}{2 \zeta}(1+\zeta)\right],
\end{aligned}
$$

( - for $n \leq 1$ and + for $n \geq 1$ ). The variation of $\lambda^{(1)}$ and $\lambda^{(2)}$ as functions of $U$ is reported in Fig. 1. Note the opening of a gap delimited by the values of $\lambda^{(2)}$ in the vacancy regime $(n \leq 1)$ and the electronic regime $(n \geq 1)$. In fact, one can demonstrate the formal identity between the Lagrange multiplier $\lambda^{(2)}$ and the chemical potential $\mu$ derived from $(-d F / d \delta)$. The observed gap of width $\Delta=U \sqrt{1-U_{c} / U}$

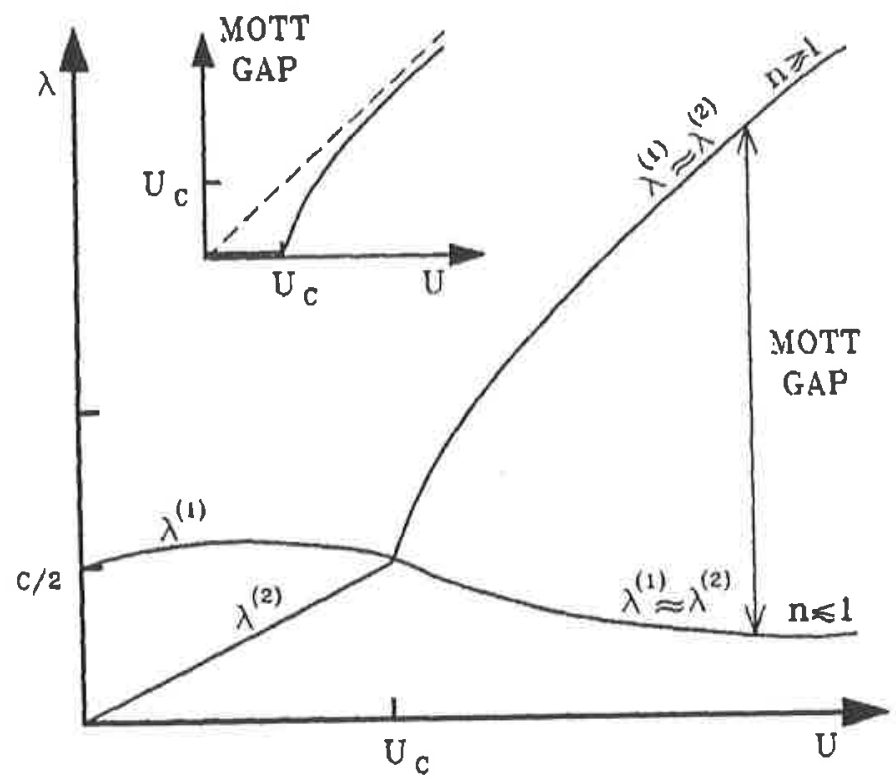

Fig. 1. Saddle-point values of the Lagrange multipliers $\lambda^{(1)}$ and $\lambda^{(2)}$ as functions of $U$. Notice the opening of a Mott gap for $U>U_{\mathrm{e}}$. In the insert is seen the $U$ dependence of the Mott gap (extracted from Ref. 24). 
(Fig. 1) is nothing but the Mott gap. Table 1 summarizes the saddle-point values of the boson fields and Lagrange multipliers in regimes I and II (up to corrections in $\delta^{2}$ ).

\section{Gaussian Fluctuations}

For the derivation of the quantum fluctuations, ${ }^{22-24}$ we will again use the diagonal representation of Kotliar-Ruckenstein which introduces two kinds of boson fields, $p_{0}$ and $p_{1 z}$, corresponding respectively to the spins $S=0$ and $S=1$. To restore the spin-rotation invariance, at least in the paramagnetic phase, we should only have to balance the weight of the $S=1$ contribution by a factor of 3 . In full generality, the slave-bosons are complex fields, the phases of which are essential to enforcing the constraints. However, it is often more convenient to operate a local gauge transformation which leaves the Lagrangian unchanged and absorb the phase of the boson fields into the Lagrangian multipliers:

Table 1. Saddle-point values of boson fields and Lagrange multipliers in regimes I and II (within the notation $\zeta=\sqrt{1-1 / u}$ ) (extracted from Ref. 24).

\begin{tabular}{|c|c|c|}
\hline & Rcgime I $\left(n=1, U<U_{\mathrm{c}}\right)$ & Regime II $\left(n<1, U>U_{\mathrm{c}}\right)$ \\
\hline$x^{2}$ & $(1-u)+\left[\frac{u}{(1-u)^{2}}-u(\alpha-1)\right] \delta^{2}$ & $\frac{\delta}{\zeta}-\frac{\delta^{2}}{2 u \zeta^{4}}$ \\
\hline$e^{2}$ & $\frac{(1-u)}{4}+\frac{\delta}{2}+\left[\frac{1}{4(1-u)^{2}}-\frac{u(\alpha-1)}{4}\right] \delta^{2}$ & $\frac{\delta}{4 \zeta}(1+\zeta)^{2}-\frac{\delta^{2}}{8 u^{2} \zeta^{4}}$ \\
\hline$d^{2}$ & $\frac{(1-u)}{4}-\frac{\delta}{2}+\left[\frac{1}{4(1-u)^{2}}-\frac{u(\alpha-1)}{4}\right] \delta^{2}$ & $\frac{\delta}{4 \zeta}(1-\zeta)^{2}-\frac{\delta^{2}}{8 u^{2} \zeta^{4}}$ \\
\hline$p^{2}$ & $\frac{(1+u)}{4}-\left[\frac{1}{4(1-u)^{2}}-\frac{u(\alpha-1)}{4}\right] \delta^{2}$ & $\frac{1}{2}-\frac{\left(1+\zeta^{2}\right)}{4 \zeta} \delta+\frac{\delta^{2}}{8 u^{2} \zeta^{4}}$ \\
\hline \multirow[t]{2}{*}{$q$} & $\left(1-u^{2}\right)+\left[\frac{2 u^{2}}{(1-u)^{2}}-1-2 u^{2}(\alpha-1)\right] \delta^{2}$ & $\frac{2 \delta}{\zeta}-\left(1+\frac{1}{\zeta^{4}}\right) \delta^{2}$ \\
\hline & $\left(1-\delta^{2}\right)$ & $\left(1-\delta^{2}\right)$ \\
\hline$\lambda^{(1)}$ & $\frac{U_{c}}{4}(1+u)(2-u)$ & $\frac{U}{2}(1-\zeta)\left[1+\frac{\delta}{\zeta}(1+\zeta)\right]$ \\
\hline$\lambda^{(2)}$ & $\frac{U}{2}$ & $\frac{U}{2}(1+\zeta)\left[1-\frac{\delta}{2 \zeta}(1+\zeta)\right]$ \\
\hline$\lambda^{(1)}-\lambda^{(2)}$ & $\frac{U_{c}}{4}(1-u)(2+u)$ & $\frac{3 U_{\mathrm{c}}}{4} \frac{\delta}{\zeta}$ \\
\hline
\end{tabular}




$$
\begin{gathered}
e_{i} \rightarrow e_{i}^{\prime} \exp \left(i \theta_{i}\right), \\
p_{i \sigma} \rightarrow p_{i \sigma}^{\prime} \exp i\left(\theta_{i}-\chi_{i \sigma}\right), \\
d_{i} \rightarrow d_{i}^{\prime} \exp i\left(\theta_{i}-\sum_{\sigma}\right)\left(\chi_{i \sigma}\right), \\
c_{i \sigma} \rightarrow c_{i \sigma}^{\prime} \exp i \chi_{i \sigma} c_{i \sigma}^{\prime}, \\
\lambda_{i}^{(1)} \rightarrow \lambda_{i}^{(1)^{\prime}}+i\left(d \theta_{i} / d \tau\right), \\
\lambda_{i \sigma}^{(2)} \rightarrow \lambda_{i \sigma}^{(2)^{\prime}}+i\left(d \chi_{i \sigma} / d \tau\right) .
\end{gathered}
$$

This defines the "radial" gauge in which the boson fields are real, and the Lagrange multipliers are no longer just time-independent variables, but are also boson fields. We are thus lead to the situation where 2 types of fermions $\left(c_{i \sigma}\right)$ are in interaction with 7 types of bosons $\left(e_{i}, p_{i \sigma}, d_{i}, \lambda_{i}^{(1)}, \lambda_{i \sigma}^{(2)}\right)$, or, more precisely, 11 types of bosons if we restore the spin-rotation invariance. Fortunately, the problem greatly simplifies if one classifies the boson fields into symmetric and antisymmetric channels, $\left\{\alpha_{\mu}, \mu=1\right.$ to 5$\}=\left\{e_{i}, d_{i}, p_{i 0}=\frac{\Sigma_{\sigma} p_{i o}}{2}, \lambda_{i}^{(1)}, \lambda_{i 0}^{(2)}=\frac{\Sigma_{\alpha} c_{i n}^{(2)}}{2}\right\}$ and $\left\{\alpha_{\mu}, \mu=6,7\right\}=\left\{p_{i z}=\frac{p_{1}-p_{i 4}}{2}, \lambda_{i z}^{(2)}=\frac{\lambda_{i i}^{(2)}-\lambda(i)}{2}\right\}$. Then, automatically, the unperturbated matricial boson propagator $\widetilde{D}_{0}^{-1}$ appears to be block-diagonal. In the paramagnetic case,

$$
\tilde{D}_{0}^{-1}=\left(\begin{array}{cc}
\tilde{D}_{0 s}^{-1} & 0 \\
0 & \tilde{D}_{0 a}^{-1}
\end{array}\right),
$$

where

$$
\widetilde{D}_{0}^{-1}=\left(\begin{array}{ccccc}
\lambda_{0}^{(1)} & 0 & 0 & e_{0} & -0 \\
0 & u+\lambda_{0}^{(1)}-\lambda_{0}^{(2)} & -0 & d_{0} & -2 d_{0} \\
0 & 0 & 2\left(\lambda_{0}^{(1)}-\lambda_{0}^{(2)}\right) & 2 p_{0} & -2 p_{0} \\
e_{0} & d_{0} & 2 p_{0} & 0 & 0 \\
0 & -2 d_{0} & -2 p_{0} & 0 & 0
\end{array}\right)
$$

and

$$
\tilde{\mathrm{D}}_{0 a}^{-1}=\left(\begin{array}{cc}
2\left(\lambda_{0}^{(1)}-\lambda_{0}^{(2)}\right) & -4 p_{0} \\
-4 p_{0} & 0
\end{array}\right)
$$

This last matrix in fact appears thrice, because of the spin-rotation invariance. Including the fluctuations, the Lagrangian can be written

$$
\mathscr{L}(\tau)=\mathscr{L}^{B}(\tau)+\mathscr{L}^{F}(\tau)
$$


with

$$
\begin{gathered}
\mathscr{L}^{B}(\tau)=\sum_{u_{v} v=1 \text { to } 7} \alpha_{\mu_{i}}^{+}\left(\widetilde{D}_{0}^{-1}\right)_{\mu v} \alpha_{v_{i}}, \\
\mathscr{L}^{F}(\tau)=\sum_{i j \sigma} c_{i \sigma}^{+}\left[\left(\partial \tau+\lambda_{i \sigma}^{(2)}\right) \delta_{i j}+t_{i j} z_{i \sigma}^{+} z_{j \sigma}\right] c_{j \sigma} .
\end{gathered}
$$

Letting the fields $\lambda_{i \sigma}^{(2)}$ and $z_{i \sigma}$ fluctuate around their saddle-point values, we have

$$
\begin{aligned}
& \mathscr{L}^{F}(\tau)=\sum_{i j \sigma} c_{i \sigma}^{+}\left[\left(\partial \tau+\lambda_{0 \sigma}^{(2)}+\delta \lambda_{i \sigma}^{(2)}\right) \delta_{i j}+t_{i j}\left(z_{0}+\delta z_{i \sigma}\right)\left(z_{0 \sigma}+\delta z_{j \sigma}\right)\right] c_{j \sigma} \\
& =\sum_{\mathbf{k q \sigma}} c_{\mathbf{k}+q \sigma}^{+}\left[\left(\partial \tau+q_{0 \sigma} \varepsilon_{\mathbf{k} \sigma}+\lambda_{0 \sigma}^{(2)}\right) \delta \delta_{q}+z_{0 \sigma} \delta z_{-\mathbf{q}, \sigma}^{+} \varepsilon_{\mathbf{k}}+z_{0 \sigma} \delta z_{\mathbf{q}, \sigma} \varepsilon_{\mathbf{k}+\mathbf{q}}\right.
\end{aligned}
$$

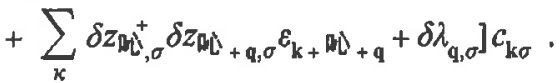

By integrating over the Grassman variables and expanding up to the second order in the boson variables, we obtain the Gaussian corrections $S^{(2)}$ to the action:

$$
\begin{aligned}
S^{(2)}= & \frac{1}{\beta} \sum_{\mu, v=-1, \ldots, 7} d \alpha_{\mu}(\mathbf{q})\left[\left(\tilde{D}_{0}^{-1}\right)_{\mu \nu}+\sum_{\mathbf{k} \sigma} A_{\mu \nu}^{\sigma}(\mathbf{k}, \mathbf{q}) G_{\mathbf{k} \sigma}\right. \\
& \left.+\sum_{k \sigma} B_{\mu}^{\sigma}(\mathbf{k}, \mathbf{q}) B_{\nu}^{\sigma}(\mathbf{k}, \mathbf{q}) G_{\mathbf{k} \sigma} G_{\mathbf{k}+\mathbf{q}, \sigma}\right] d \alpha_{\nu}(\mathbf{q}) \\
= & \frac{1}{\beta} \sum_{\mathbf{q}, \omega_{\mu, \nu}} d \alpha_{\mu}(\mathbf{q}) \tilde{D}^{-1}\left(q, \omega_{\nu}\right) d \alpha_{v}(\mathbf{q})
\end{aligned}
$$

with

$$
\begin{aligned}
A_{\mu \nu}^{\sigma}(\mathbf{k}, \mathbf{q}) & =z_{0} \varepsilon_{k} \frac{\partial^{2} z_{\sigma}}{\partial \alpha_{\mu} \partial \alpha_{v}} \delta_{q}+\varepsilon_{k+q} \frac{\partial z_{\sigma}}{\partial \alpha_{\mu}} \frac{\partial z_{\sigma}}{\partial \alpha_{\nu}} \text { if } \mu, v=1,2,3 \text { or } 6, \\
& =0 \quad \text { if } \mu, v=4,5 \text { or } 7 . \\
B_{\mu}^{\sigma}(\mathbf{k}, \mathbf{q}) & =z_{0}\left(\varepsilon_{\mathbf{k}}+\varepsilon_{\mathbf{k}+q}\right) \frac{\partial z_{\sigma}}{\partial \alpha_{\mu}} \text { if } i, j=1,2,3 \text { or } 6, \\
& =\frac{\partial \lambda_{\sigma}^{(2)}}{\partial \alpha_{\mu}} \text { if } i=5 \text { or } 7, \\
& =0 \text { if } i=4 .
\end{aligned}
$$


By integrating over the bosonic variables, one gets the contribution to the free energy:

$$
\begin{aligned}
F^{(2)} & =\frac{1}{\beta} \sum_{\mathbf{q}, \omega_{r}} \operatorname{Ln}\left[\operatorname{det} . \widetilde{D}^{-1}\left(\mathbf{q}, \omega_{v}\right)\right] \\
& =\frac{1}{\beta} \sum_{\mathbf{q}, \omega_{v}} \operatorname{Ln}\left[\operatorname{det} .\left(\widetilde{D}_{1}^{-1}\left(\mathbf{q}, \omega_{\nu}\right)\right)+\sum_{\mathbf{k}, \omega_{n}} \tilde{\Gamma}(\mathbf{k}+\mathbf{q}) G\left(\mathbf{k}, \omega_{n}\right) G\left(\mathbf{k}+\mathbf{q}, \omega_{n}+\omega_{\nu}\right)\right],
\end{aligned}
$$

where, on the basis of $\left\{\alpha_{\mu}\right\}, \widetilde{D}_{1}^{-1}$ and $\tilde{\Gamma}$ are both block-diagonal. The role of the quantum fluctuations is to introduce some effective interactions (mediated by the slave-bosons) among the quasiparticles defined at the saddle-point level. Coming from the structure of the boson fields, there is a complete separation between symmetric and antisymmetric channels as represented on the energy diagram of Fig. 2. This is the great advantage of working in the $N=2$ slave-boson representation since the bubble diagram and the ladder diagram (the latter being essential to describing the magnetism) appear at the same level of the expansion. ${ }^{26}$ This is to be compared with the limit $U=\infty$, large $N$, where only one slave-boson $e$ is required, and the gaussian fluctuations (of order $1 / N$ ) lead only to the bubble contribution, and so miss the ladder or magnetic contribution (which only appears at the following order $1 / N^{2}$ ). Of course, the saddle-point is no longer exact as in the $N=\infty$ limit, and there is no longer a small parameter such as $1 / N$ to control the expansion. However, this apparent loss of accuracy is compensated by the gain in the physical meaning of the expansion since now the ladder (spin-fluctuation) contribution is already present at the level of the gaussian fluctuations.

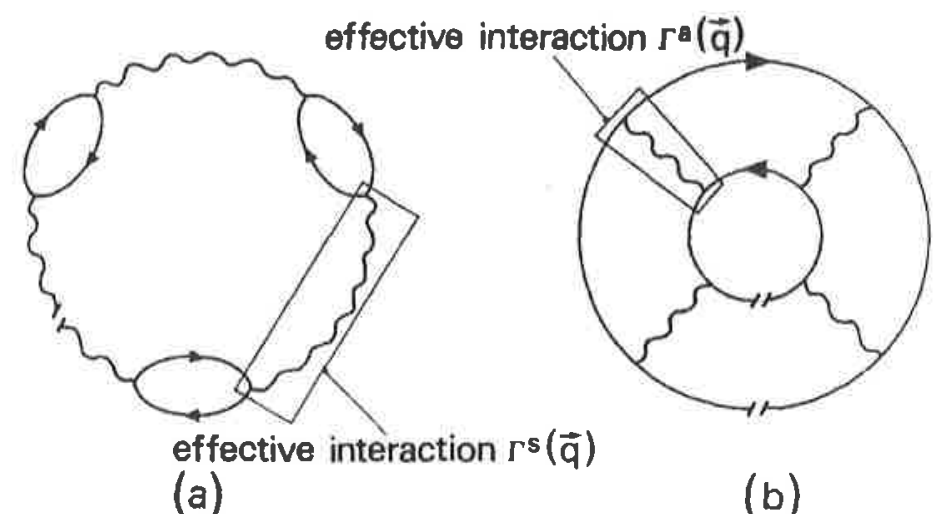

Fig. 2. Diagrammatic representation of the free cnergy bubble diagram (2a), ladder diagram (2b). 
When only the linear term is kept (random phase approximation), we have

$$
\begin{aligned}
F^{(2)}= & \frac{1}{\beta} \sum_{\mathbf{k}, \omega_{v}} \operatorname{Ln}\left[1+\sum_{\mathbf{k}, \omega_{n}} \operatorname{Tr} \cdot\left\{\tilde{D}_{1 s}(\mathbf{q}) \tilde{\Gamma}_{s}(\mathbf{k}+\mathbf{q})_{-}\right\} G\left(\mathbf{k}, \omega_{n}\right) G\left(\mathbf{k}+\mathbf{q}, \omega_{n}+\omega_{v}\right)\right] \\
& +3 \operatorname{Ln}\left[1+\sum_{\mathbf{k}, \omega_{n}} \operatorname{Tr} \cdot\left\{\widetilde{D}_{1 a}(\mathbf{q}) \widetilde{\Gamma}_{a}(\mathbf{k}+\mathbf{q})\right\} G\left(\mathbf{k}, \omega_{n}\right) G\left(\mathbf{k}+\mathbf{q}, \omega_{n}+\omega_{v}\right)\right] .
\end{aligned}
$$

This provides the microscopic basis for a Fermi liquid description with quasiparticles interacting through some effective interactions mediated by the symmetric and antisymmetric part of the slave bosons. Within the approximation where one can neglect the $\mathbf{k}$ dependence of $\Gamma_{s(a)}(\mathbf{k}, \mathbf{q})$, the Landau parameters are simply obtained by expanding the effective interactions into Legendre polynomials (or cubic harmonics) if the symmetry is spherical (or cubic).

Typically in the $l=0$ channel, one obtains

$$
F_{0}^{a}=\left[-1+\frac{e d}{(e+d)^{2}} \frac{1}{\left(2 p^{2}\right)^{2}}\right] \alpha^{-1},
$$

i.e.

$$
\begin{gathered}
F_{0}^{a}=\left[-1+\frac{1}{(1+u)^{2}}\right] \alpha^{-1} \text { and } F_{0}^{s}=\left[-1+\frac{1}{(1-u)^{2}}\right] \alpha^{-1}, \\
\text { (in regime I) } \\
F_{0}^{a}=\left[-1+\frac{1}{4 u}\right] \alpha^{-1} \text { and } F_{0}^{s}=\left[-1+\frac{2 u-1}{2 \delta \sqrt{u(u-1)}}\right] \alpha^{-1} .
\end{gathered}
$$

(in regime II)

It is remarkable that the expressions found for $F_{0}^{\text {s(a) }}$ via the gaussian fluctuations (brute force method) coincide with those usually obtained within the Gutzwiller approximation. This can be easily understood if one realizes that the Gutzwiller approximation is in fact a kind of "self-consistent" mean-field treatment with an implicit dependence on the parameter $q_{0 \sigma}$ (that is to say, of the saddle-point values of the boson fields) with the external excitation. However, it is then clear that the slave-boson approach goes beyond the Gutzwiller approximation, since

(i) the Landau parameter can also be defined in the following channel $l \neq 0$ (while being automatically neglected in the GA);

(ii) the dynamic effects which were missing in the Gutzwiller approximation are now clearly incorporated through Eq. (3.7); 
(iii) the extension to finite temperatures is now possible, which avoids the delicate question of determining the entropy in the Gutzwiller approximation.

Within the approximation in which one can neglect the $k$-dependence of $\Gamma_{s}(\mathbf{k}, \mathbf{q})$ and only keep the contribution of the first main channel $l=0$, one gets

$$
\begin{aligned}
F^{(2)}= & \frac{1}{2 \pi} \sum_{q} \int_{-\infty}^{+\infty} d \omega \operatorname{coth} \frac{\beta \omega}{2}\left\{\operatorname{Im} \cdot \operatorname{Ln}\left[1+F_{0}^{s} \chi_{0}\langle\mathbf{q}, \omega)\right]\right. \\
& \left.+3 \operatorname{Im} \cdot \operatorname{Ln}\left[1+F_{0}^{a} \chi_{0}(\mathbf{q}, \omega)\right]\right\} .
\end{aligned}
$$

In the weak-coupling regime, $F_{0}^{s}=-F_{0}^{a}=2 u \alpha^{-1}$, and one recovers the standard results of the random-phase approximation. Thus, this approach provides a unique interpolation between the weak coupling regime (RPA) and the strong coupling regime, incorporating the dynamic effects around the Gutzwiller solution.

\section{Physical Implications}

The above analysis provides the microscopic basis for a Fermi-liquid theory. It allows for the determination of the Landau parameters. From this, one can deduce a series of physical implications, all of them being typical of a Fermi liquid.

(i) The dynamic correlation functions are represented diagrammatically in Fig. 3. Their analytical expressions ${ }^{23,24}$ are given by

(a)

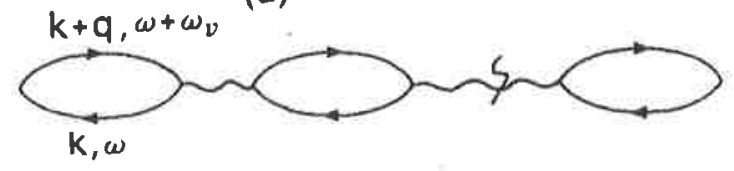

(b)

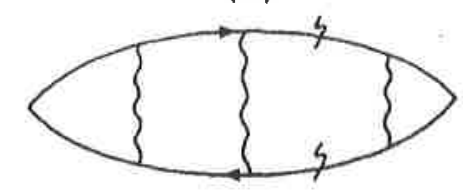

Fig. 3. Density-density and spin-spin correlation functions. 


$$
\begin{aligned}
\kappa(\mathbf{q}, \omega) & =\frac{\chi_{0}(\mathbf{q}, \omega) q_{0}^{-1}}{1+F_{0}^{s} \chi_{0}(\mathbf{q}, \omega) / \chi_{0}}, \\
\chi_{s}(\mathbf{q}, \omega) & =\frac{\chi_{0}(\mathbf{q}, \omega) q_{0}^{-1}}{1+F_{0}^{a} \chi_{0}(\mathbf{q}, \omega) / \chi_{0}}, \\
\chi_{c}(\mathbf{q}, \omega) & =0 .
\end{aligned}
$$

The charge susceptibility is strictly zero, as in any RPA calculation. It is due to the decoupling between the different modes of fluctuations. This clearly appears in the block-diagonal structure of the boson-propagator matrix $D$. The first block diagonal $(s)$ connected to $\left(\Sigma_{s} n_{s}\right)$ is related to the density-density correlation, and the second part $(a)$ connected to $\left(n_{\uparrow}-n_{\downarrow}\right)=m_{z}\left(m_{x}\right.$ or $\left.m_{y}\right)$ is related to the spin-spin correlations. Again, for $q=\omega=0$, one recovers the result of the Gutzwiller approximation.

In regime II, $F_{0}^{s}=1 / \delta$, which just compensates the effect of the renormalization factor $q_{0}^{-1}$. It gives an unenhanced compressibility as expected for an incompressible Fermi liquid.

The discussion of the magnetic instabilities essentially depends on the symmetry considered. In the spherical case ( $\chi_{0}$ given by the Lindhard functions), one finds a ferromagnetic instability at large $U$ but no antiferromagnetic instability. The situation is rather different in the case of an alternating structure (e.g. simple cubic) where the nesting property of the paramagnetic Fermi surface gives an antiferromagnetic instability at $n=1$ for an infinitesimally small value of $U$. The corresponding phase diagram obtained in Ref. 10 is reported in Fig. 4. It is easy to check that the ferromagnetic instability occurs above $U_{F}=1 /[4(1-\alpha)]$ at $n=1$. The ferromagnetic-antiferromagnetic boundary is asymptotic to the line $n=1$ at $U=\infty$ as required by Nagaoka's theorem. ${ }^{33}$ The disappearance of ferromagnetism at large $U$ for $1-\mathrm{n}=\delta=0.38$ agrees qualitatively with the result of Kanamori. ${ }^{34}$

(ii) From the expression (3.9) of the free energy, one can also get a $T^{3} \operatorname{Ln} T$ contribution to the specific heat in complete analogy with what occurs in paramagnon theory. ${ }^{35.36}$ By using the Lindhard functions for the bare susceptibilities (free electrons)

$$
\chi_{0}(q, \omega)=\rho\left[1+\frac{1}{12} \frac{q^{2}}{k_{\mathrm{F}}^{2}}+i \frac{\pi}{2} \frac{\omega}{q v_{\mathrm{F}}}\right]=\chi^{\prime}{ }_{0}+i \chi^{\prime \prime}{ }_{0}
$$

and expanding

$$
\operatorname{lm} \cdot \operatorname{Ln}\left[1+F_{0} \chi_{0}(q, \omega)\right]=\operatorname{Arctg} \frac{F_{0} \chi^{\prime \prime}{ }_{0}}{1+F_{0} \chi_{0}^{\prime}}
$$




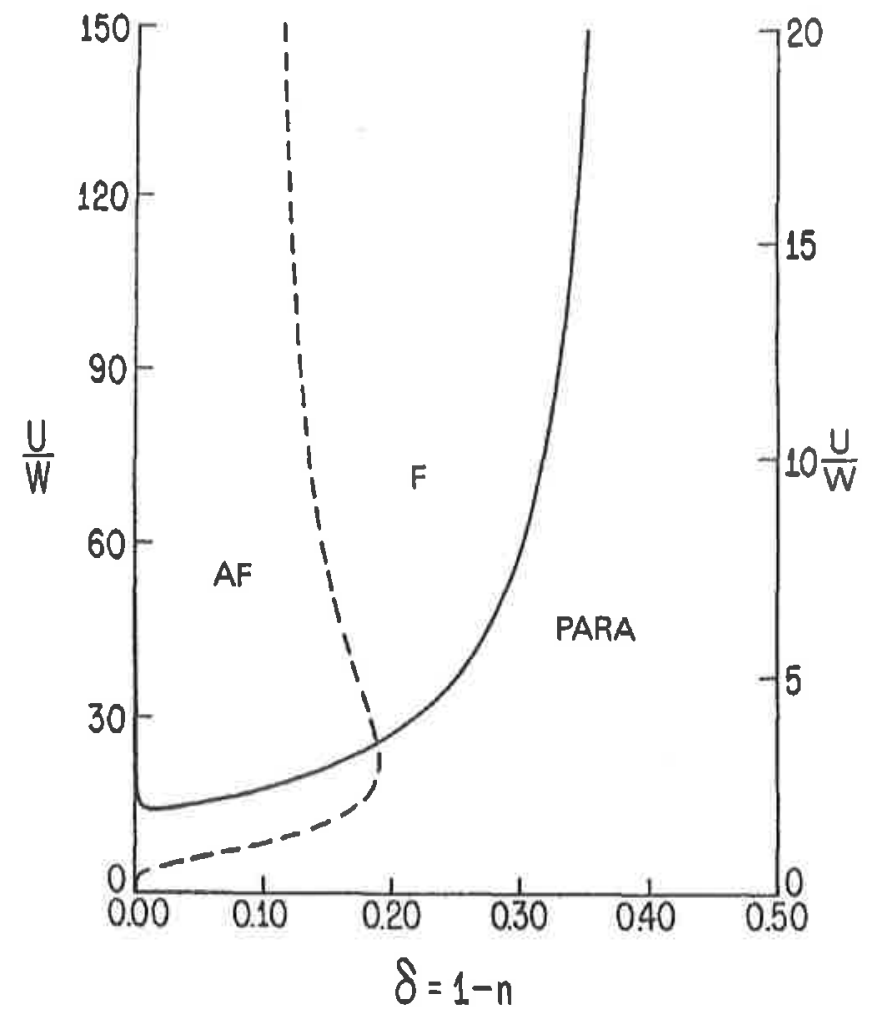

Fig. 4. Magnetic phase diagram (cf. Ref, 10).

to third order in $\omega / q v F$, one obtains a $T^{4} \operatorname{Ln} T$ contribution to the free energy. The resulting specific heat thus has a $T^{3} L n T$ behaviour

$$
\begin{aligned}
\Delta C & =\Delta C_{s}+\Delta C_{a}, \\
\Delta C_{q(s)} & =\frac{9 \pi^{4}}{40} \frac{n}{\left(T_{\mathrm{F}}^{*}\right)^{3}}\left(A_{0}^{a(s)}\right)^{2}\left(1-\frac{\dot{\pi}^{2}}{12} A_{0}^{a(s)}\right) T^{3} \operatorname{Ln} \mathrm{T},
\end{aligned}
$$

where $A_{0}^{a(s)}$ is the quasiparticule scattering amplitude related to $F_{0}^{a(s)}$ by

$$
A_{0}^{a(s)}=\frac{F_{0}^{a(s)}}{1+F_{0}^{a(s)}},
$$

$n$ is the number of particles $=k_{\mathrm{F}}^{3} / 3 \pi^{2}$ and $T_{\mathrm{F}}^{*}$ is the renormalized Fermi temperature $\left(T_{\mathrm{F}}^{*}=T_{F} q_{0}\right)$. 
It should be noted that generally the contribution coming from the spin fluctuation $\left(\Delta C_{a}\right)$ is larger by at least two orders of magnitude than the density contribution $\left(\Delta C_{s}\right)$. This result on the $T^{3} L n T$ term contribution to the specific heat was first obtained by Rasul and $\mathrm{Li}^{22}$ in the weak-coupling regime without nesting of the Fermi surface, to avoid the antiferromagnetic instability. It was applied with some success to $\mathrm{He}^{3}$. However, the coefficient found differed by a factor of three from the standard calculation of Pethick and Carneiro, ${ }^{36}$ performed in the frame of a Fermi liquid theory. It was soon remarked ${ }^{25}$ that this discrepancy was due to the spin-rotation symmetry breaking of the original KR representation. It is automatically corrected if one introduces the generalized SRI representation. When the localization edge is approached $\left(U \rightarrow U_{\mathrm{c}}\right), T_{F}^{*}=T_{F}\left(\mathrm{~m} / \mathrm{m}^{*}\right)$ goes to zero while $A_{0}^{a}$ remains finite $(=-0.75)$. Thus one expects a huge contribution to the $T^{3} \operatorname{Ln} T$ term.

More generally, this $T^{3} \operatorname{Ln} T$ term can be regarded as the beginning of a crossover between the low temperature regime where the specific heat is linear in $T$, and the high temperature regime exhibiting a slow drift in $T L n T$.

(iii) The eventual superfluid instability of the model can be examined by looking at the electron-electron (Cooper) correlation function. The Bethe-Salpeter equation for the correlation function is written in decoupled channels $l$. The vertex involved is nothing but the scattering amplitude $A_{\%}^{\prime \prime}$. A superfluid instability of type $l$ is obtained each time the scattering amplitude $A_{l}^{a}$ derived from microscopic basis is negative. It is in this way that a $d$-wave superconductivity $\left(A_{i=2}^{a}<0\right)$ has been predicted for the $1 / N$ slave-boson expansion of the Anderson lattice $\operatorname{model}^{9}$ (heavy fermion). When applied to the Hubbard model, a $p$-wave superfluid instability $\left(A_{l=1}^{a}<0\right)$ has been found ${ }^{37}$ in regime I near the localization edge. For a given value of the cutoff $\left(T_{F}^{*}\right)$, the results obtained for $T_{\mathrm{c}}$ are in reasonably good agreement with experiments on liquid $\mathrm{He}^{3}$ under pressure.

\section{Conclusion}

The slave-boson representation of the Hubbard model discussed in this paper appears to provide a very interesting starting point to study strongly-correlated fermion systems. It is proved to be equivalent to the Gutzwiller approximation at the saddle-point level in its self-consistent version. A Mott transition (Brinkman-Rice) is expected at this level for any dimensionality. One of its manifestation is the splitting in the values of the Lagrange multiplier $\lambda^{(2)}$ on each side of half-filling (Mott-Hubbard gap). The undeniable interest of the approach is certain to allow for a systematic expansion beyond the Gutzwiller approximation. This is proved to restore the dynamic effects which were missed in the so-called "almost" localized model provided by the GA. It is remarkable that the charge fluctuations (bubble diagrams) and the spin fluctuations (ladder diagrams) appear at the same level of the gaussian fluctuations. This is to be compared with the $1 / N$ expansion, which only gives the charge fluctuations and misses the spin 
fluctuations. We have stressed how this approach provides the miscroscopic basis of a Fermi liquid theory, in which the Landau parameters are evaluated from the initial parameters $U, W, n$.

We have essentially focused on the long wavelength, low frequency limit leading to the usual behaviour of a Fermi liquid (dynamic correlation functions, $T^{3} L n T$ contribution to the specific heat, superfluid interaction...). It would of course be of great interest to consider the other Fermi-liquid properties of these systems (transport coefficients, viscosity, thermal conductivity, scattering amplitude ...). All of this concerns the low temperature regime characterized by Fermi liquid behaviour. Another interesting question is to examine the crossover between the low temperature Fermi liquid regime, and the classical high-temperature limit. This is likely to be governed by interacting fluctuations, and the hope is that it could be described by an appropriate "self-consistent" fluctuation theory. As for the magnetic instabilities, only structures have been considered until now (ferromagnetic, AF, paramagnetic). But it is clear that the approach could also be extended to the study of any more sophisticated instabilities (spiral, ${ }^{38}$ incommensurable magnetic structures...).

Another promising aspect concerns the possibility of getting rid of the Fermi liquid behaviour when the dimensionality is lowered. Instead of starting from a Fermi gas (characterized by the Lindhard functions $\chi_{0}$ ) such as that in the GWF, one can imagine starting from a BCS type wave function. After projecting out doubly occupied sites, this will give a description of what is called RVB (resonant valence bond) state, ${ }^{4}$ differing from a true BCS state by an unlocked phase, since the number of particles is kept fixed. Another issue is the possibility of an orbital ferromagnetic symmetry breaking, as occurs in the commensurable flux phase. ${ }^{39-41}$ This is characterized by a gauge field whose flux per unitary plaquette is non-zero connected to the number of particles. Here again, the slaveboson approach constitutes the ideal framework in which to discuss the stability of the different states and their corresponding properties.

\section{Acknowledgments}

It is a pleasure to thank D. Grempel, P. Nozières, J. P. Rodriguez and N. Schopohl for very stimulating and useful discussions. I also acknowledge the hospitality of the ISI (Internationai Scientific Interchange) of Torino, Italy.

\section{References}

1. P. W. Anderson and W. Brinkman, The Physics of Liquid and Solid Helium, Part II, cds. K. H. Bennemann and J. B. Ketterson, (Wiley, 1978).

2. P. A. Lee, T. M. Rice, J. W. Serene, L. J. Sham, and J. W. Wilkins, Comments Solid State Phys. 12 (1986) 99.

3. R. E. Prange and M. G. Girvin, The Quantum Hall Effect, (Springer-Verlag, 1987).

4. P. W. Anderson, in Frontiers and Boderlines in Many-Particle Physics, International School of Physics "Enrico Fermi", Course C IV, eds. R. A. Broglia and J. R. Schrieffer, (North-Holland, 1988). 
5. P. Coleman, Phys. Rev. B29 (1984) 3035.

6. N. Read and D. Newns, J. Phys. C16 (1983) 3273.

7. A. J. Millis and P. A. Lee, Phys. Rev. B35 (1987) 3394.

8. A. Auerbach and K. Levin, Phys. Rev. Lett. 57 (1986) 877.

9. M. Lavagna, A. J. Millis, and P. A. Lee, Phys. Rev. Lett. 58 (1987) 266.

10. G. Kotliar and A. E. Ruckenstein, Phys. Rev. Lett. 57 (1986) 1362.

11. M. C. Gutzwiller, Phys. Rev. Lett. 10 (1963) 159.

12. M. C. Gutzwiller, Phys. Rev. 134 (1964) A923; 137 (1965) A 1726.

13. D. Vollhardt, Rev. Mod. Phys. 56 (1984) 99.

14. P. Noziercs, Cours au College de France: Magnetism and Localization in Fermi liquids, 1986 (unpublished).

15. W. F. Brinkman and T. M. Rice, Phys. Rev. B2 (1970) 4302.

16. N. F. Berk and J, R. Schrieffer, Phys. Rev. Lett 17 (1966) 433.

17. S. Doniach and S. Engelsberg, Phys. Rev. Lett. 17 (1966) 7502.

18. W. Metzner and D. Vollhardt, Phys. Rev. Lett. 59 (1987) 121; Phys. Rev. B37 (1988) 7382 .

19. F. Gebhard and D. Vollhardt, Phys. Rev. Lett. 59 (1987) 1472; Phys. Rev. B38 (1988) 6911.

20. F. D. M. Haldane, Phys. Rev. Lett. 60 (1988) 635.

21. B. S. Shastry, Phys. Rev. Lett. 60 (1988) 639.

22. J. W. Rasul and T. Li, J. Phys C21 (1988) 5119.

23. T. C. Li and J. W. Rasul, Phys. Rev. B39 (1989) 4630.

24. M. Lavagna, Phys. Rev. B41 (1990) 142.

25. T. Li, P. Wölfle and P. J. Hirschfeld, Phys. Rev. B40 (1989) 6817.

26. M. Lavagna, Helvetica Phys. Acta 63 (1990) 310.

27. G. Kotliar and J. Liu, Phys. Rev. Lett. 61 (1988) 1784.

28. K. Seiler, C. Gros, T. M. Rice, K. Ueda, and D. Vollhardı, J. Low Temp. Phys. 64 (1986) 195

29. A. Houghton, N. Read and H. Won, Phys. Rev. B37 (1988) 3782.

30. D. P. Arovas and A. Aucrbach, Phys, Rev, B38 (1988) 316.

31. P. A. Lee, Phys. Rev. Lett. 63 (1989) 680.

32. D. Vollhardt, P. Wölfle and P. W. Anderson, Phys, Rev. B35 (1987) 6703.

33. Y. Nagaoka, Phys. Rev. 147 (1966) 392.

34. J. Kanamori, Prog. Theor. Phys. 30 (1964) 257.

35. W. Brenig, H. J. Mikeska and E. Riedel, Z. Phys. 206 (1967) 439.

36. C. J. Pethick and G. M. Carneiro, Phys. Rev. A7 (1973) 304.

37. J. W. Rasul, T. Li and H. Beck, Phys. Rev. B39 (1989) 4191.

38. B. I. Shraiman, and E. D. Siggia, Phys. Rev. Lett. 62 (1989) 1564.

39. I. Affleck and J. B. Marston, Phys. Rev. B37 (1988) 3774.

40. P. W. Anderson, B. S. Shastry and D. Hristopoulos, Phys. Rev. B40 (1989) 8939.

41. P. Lederer, D. Poilblanc and T. M. Rice, Phys. Rev. Lett. 63 (1989) 907. 